\title{
PLANIFICACIÓN REGIONAL Y URBANA: UN ENFOQUE INTEGRADO ESPACIO - TIEMPO
}

HAROLD BANGUERO LOZANO

Universidad Autónoma de Occidente,Cali, Colombia

\section{RESU MEN}

Los ejercicios de planificación se hacen generalmente en una sola dimensión, el tiempo o el espacio. Los planes de desarrollo, en los cuales se definen objetivos, metas, programas y proyectos a ejecutar en un horizonte de tiempo determinado y los de ordenamiento territorial, en los cuales se definen los usos de los suelos y la localización de las diferentes intervenciones humanas a realizar para garantizar un desarrollo territorialmente ordenado de una región o localidad, se elaboran generalmente, sin que exista un mínimo de alineación entre los dos ejercicios. El propósito de este trabajo es presentar una propuesta de planificación en la cual se integran estas dos dimensiones aplicando dos principios fundamentales, ir del largo al corto plazo en la dimensión tiempo y del macro al micro territorio en la dimensión espacial. Posteriormente, se presenta una aplicación al caso del municipio de Buenaventura, en la región Pacífico colombiana. Se espera con ello, hacer una contribución al mejoramiento de la planificación regional y urbana en países en desarrollo.

Palabras clave: Planificación, desarrollo, tiempo, espacio, región, localidad.

Clasificaciones JEL: R12, R14, R52, R58, O21.

a Correo electrónico: hbanguero@hotmail.com. Recibido: 19 de febrero de 2019; aceptado: 25 de octubre de 2019.

Econamía\& Región Vd. 13, Nb. 2, (Cartagena, dicientre2019), pp 147- 176 


\section{ABST RACT}

\section{Urban and Regional Planning: An Integrated Space-Time Approach}

Planning exercises are often done taking into account only one dimension, either time or space. Development plans usually define objectives, goals, programs and projects considering only the time dimension, while territorial ordering plans define soil uses and location of human interventions in the space dimension, without assuring a minimum of consistency between the two exercises. The purpose of this paper is to present a proposal for planning in which these two dimensions, space and time, are fully integrated, applying two fundamental principles: to go always from the long to the short run in the time dimension and from the macro to the micro areas in the space dimension. The approach is applied to the case of Buenaventura, a municipality in the Pacific region of Colombia. It is expected that the exercise will contribute to improve regional and urban planning in developing countries.

Key words: Planning, development, time, space, region, locality.

\section{INTRODUCCIÓN}

En la tradición de la planificación regional y urbana existen dos tipos de ejercicios: los planes de desarrollo, en los cuales se definen objetivos, metas, programas y proyectos a ejecutar en un horizonte de tiempo determinado, y los planes de ordenamiento territorial, en los cuales se definen los usos de los suelos y la localización de las diferentes intervenciones humanas a realizar para garantizar el desarrollo territorialmente ordenado de una determinada región o localidad.

Por un lado, en la literatura sobre el tema se establece que los dos enfoques, de planeación en el tiempo y de planeación en el espacio, se desarrollaron de forma claramente independiente a lo largo de la historia del pensamiento económico. En efecto, desde el siglo XIX la economía espacial avanzó sustancialmente, en un camino iniciado por los trabajos pioneros de Von Thunen (1910), continuado por sus seguidores de escuela alemana y, posteriormente, en el siglo XX prosiguió su desarrollo en el mundo anglosajón. Este avance se dio hasta el punto que, en la actualidad, este tipo de planificación ha alcanzado un alto grado de elaboración tanto conceptual como empírica (Losch, 1957; Isard, 1956; 
Perroux, 1964; Matarrese, 1969; Krugman, 1991; Boisier, 1999; y Fujita, 2000).

Por otro lado, el enfoque de la planificación estratégica inicialmente propuesta por la Escuela Francesa a mediados del siglo XX (De Jouvenel, 1964; Berger,1964), también ha alcanzado un gran desarrollo en décadas recientes (Sallenave, 1995; Godet, 1985, 1995, 1997; Mojica, 1991), desplazando casi completamente a la escuela tradicional de planificación, circunscrita a ejercicios de corto plazo, basados en análisis estadísticos y proyecciones tendenciales.

La evolución separada que se ha descrito de los dos enfoques a lo largo de la historia refuerza el hecho de que, tanto en la normatividad como en la práctica, estos ejercicios se hacen de manera independiente y, hasta donde conocemos, no aparecen esfuerzos de integrarlos, a pesar de lo estrechamente relacionados que están.

En el caso colombiano ocurre algo similar, dado que la ley 152 de 1993 (Congreso de la República de Colombia, 1993) definió la normatividad sobre la elaboración de los planes de desarrollo departamentales y municipales, mientras que la ley 388 de 1997 (Congreso de la República de Colombia, 1997), estableció las normas para la elaboración de los planes de ordenamiento territorial de estas entidades territoriales. Los ejercicios de planificación se hacen de forma separada, dado que el plan de desarrollo tiene un horizonte de cuatro años -el período de una administración- en tanto que el de ordenamiento territorial tiene uno de doce -con posibilidades de ser modificado durante ese tiempo- si la administración de turno lo estima conveniente. Además, los mandatarios departamentales y municipales se limitan a hacer cumplir ambas normas, mediante ejercicios independientes que podría decirse que no reparan en la relación existente entre el espacio y el tiempo en los procesos de desarrollo regional y local.

Los dos ejercicios, sin embargo, la planeación del desarrollo en el tiempo y la planificación en el espacio, están profundamente interrelacionados y, por lo tanto, requieren definir una metodología que permita realizarlos de manera integrada y no de forma aislada, de tal forma que se garantice un mínimo de alineación entre los objetivos, metas, programas y proyectos de los planes de desarrollo y su localización en el espacio regional o local a lo largo del tiempo.

El propósito de esta monografía es conceptualizar y definir un procedimiento para alinear plenamente los ejercicios de los planes de desarrollo, formulados en un horizonte de tiempo de corto plazo (cuatro años, en el caso colombiano) y su localización en el espacio regional o local, reflejado en el POT en un horizonte de mediano plazo (12 años en el caso colombiano). Con ello se espera hacer 
una contribución al mejoramiento de la planificación regional y urbana en un contexto como el colombiano.

\section{CONCEPTOS,PRINCIPIOS Y CRITERIOS EN EL PROCESO DE LA PLANEACIÓN ESTRATÉGICA TERRITORIAL}

Los ejercicios de planificación del desarrollo persiguen objetivos de bienestar económico y social para una población, en un determinado horizonte de tiempo (Godet, 1995). Sin embargo, el logro de estos objetivos depende críticamente de las características del espacio en donde se hace la intervención, de tal forma que el desarrollo de la planificación en el tiempo queda subordinada a las restricciones que impone el territorio. En últimas, sólo serán alcanzables aquellas metas que guarden consistencia con las posibilidades que ofrece el territorio, sobre todo cuando tengan un horizonte de tiempo de corto plazo, por ser inamovibles, en tanto que en el mediano o largo plazo pueden modificarse, como producto de las intervenciones humanas (Fujita, 2000).

De manera similar, los ejercicios de planificación en el espacio local suelen desconocer los condicionamientos que impone el entorno regional en la planificación de ciudades y centros poblados. Así como el territorio puede restringir la planificación de procesos de desarrollo en el tiempo, las condiciones del entorno crean restricciones sobre la planificación de los centros urbanos poblados. Su desconocimiento puede hacer inviables soluciones que parecerían óptimas al ser contempladas en un contexto puramente local. Estas reflexiones han llevado a varios autores al desarrollo del concepto de ciudad región como eje fundamental de los procesos de planificación de los centros urbanos poblados (Matarrese, 1969; Boisier, 2006; CAF, 2010).

Con base en estas consideraciones, se han subrayado dos principios básicos, tanto desde la literatura de planificación en el tiempo (Mojica, 1991; Godet, 1995, Ortegón y Medina, 1997, entre otros), como en la del espacio (Loch, 1967; Isard, 1956; Fujita, 2000, entre otros), los cuales constituyen el punto de partida para una adecuada alineación en el tiempo y en el espacio de los dos tipos de planificación mencionados.

El primer principio consiste en que la planeación en el tiempo va del largo al corto plazo. Esto quiere decir que el proceso parte de definir una visión, objetivos y metas de largo plazo, los cuales en una etapa posterior se desagregan en metas parciales de corto plazo. La racionalidad de este principio radica en la duración de los períodos de maduración que tienen los procesos de desarrollo 
económico y social. En este sentido, el plan de largo plazo guía la elaboración de los planes de corto plazo, los cuales hacen aportes parciales al logro de los objetivos plasmados en una visión de largo plazo. Por lo tanto, si estos planes no están alineados con el de largo plazo, no está garantizado el logro del objetivo final. De esta forma, las visiones de largo plazo guían las acciones en el corto plazo y no al contrario. Este planteamiento se resume en la siguiente frase: la suma de los objetivos de corto plazo no necesariamente equivale al logro del objetivo de largo plazo, a menos que deliberadamente estén alineados para ello (Banguero, 1982, 1985; y Godet, 1995) (Gráfico 1).

\section{GRÁFICO 1}

Planificación del desarrollo en las dimensiones: espacio - tiempo

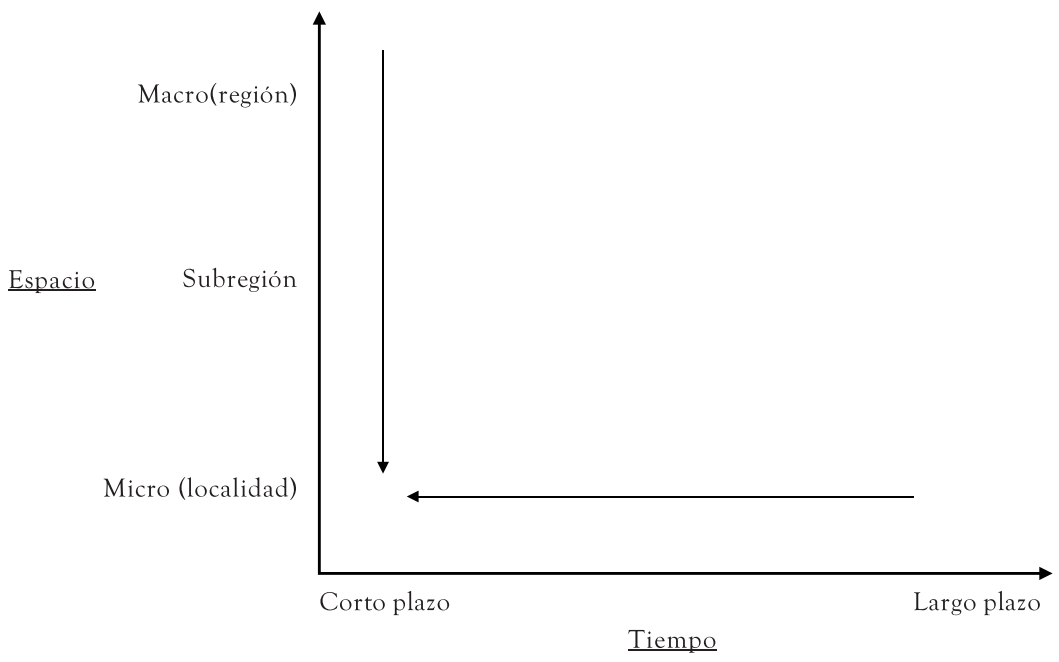

Fuente: Elaboración del autor.

El segundo principio consiste en que la planeación en el espacio va de lo macro a lo micro. Es decir, el proceso parte de la visualización de un territorio en su totalidad para luego desagregarlo en subregiones, localidades, corregimientos, comunas, barrios, etc., según sea necesario (Hincapié y Hoyos, 1990). La racionalidad de este principio radica en la interdependencia que hay entre un territorio y su entorno. En efecto, una parte importante de los fenómenos naturales que afectan un micro territorio no son controlables en su interior y, por lo tanto, requieren de intervenciones por fuera del mismo. 
Es el caso de la disponibilidad de agua, la cual con frecuencia sólo se garantiza mediante acciones en las cuencas hidrográficas localizadas en el entorno, a veces distante. Esta realidad hace que el proceso de planificación territorial se inicie en el macro territorio de tal forma que los planes a nivel micro (región o localidad) tengan en cuenta los lineamientos definidos en el macro plan (regional) de ordenamiento del territorio. El éxito de la planificación territorial dependerá críticamente de qué tan alineadas estén las acciones en los micro territorios con los lineamientos de un plan macro de ordenamiento del territorio, y de qué tanto respondan a su lógica, en el entendido de que el agregado de micro planes de ordenamiento territorial no necesariamente implica un adecuado ordenamiento de un macro territorio (Matarrese, 1969) (Gráfico 1).

Otros criterios adicionales definidos para la elaboración de este tipo de ejercicios son los definidos por un equipo de investigadores expertos en ordenamiento regional en regiones y municipios de América Latina (Hincapié y Hoyos, 1990):

1. Se trabaja la región o localidad como una totalidad que incluye zonas tanto urbanas y rurales, dado que constituye, por definición, la unidad básica de planificación.

2. El ejercicio se realiza sobre planos o mapas de la región o localidad que contengan los principales hitos geográficos que la caracterizan. Estos mapas deben contener, adicionalmente, los territorios circunvecinos de la región o localidad a planificar, con los cuales existan vínculos de conurbación, conmutación o interrelación, en una secuencia que va de la macro a la micro planificación y no al revés.

3. Se debe iniciar con la planificación del territorio en el largo plazo, describiendo la situación actual del uso del territorio y la imagen futura deseada de la región o la localidad al final del horizonte de planificación, a partir de la cual se derivan posteriormente planes de desarrollo de corto y mediano plazo. Esto refuerza, entonces, la idea de que la visión, los objetivos y las metas de estos planes se derivan del ejercicio previo de planificación del territorio a largo plazo. Las metas intermedias están alineadas con los objetivos del plan estratégico de largo plazo.

4. El ejercicio requiere de la participación de los representantes de las comunidades que habitan el territorio a planificar, para lograr un alto grado de pertinencia, compromiso y apropiación.

5. Se deben tener en cuenta las normas y directrices establecidas en el orden nacional con respecto a la planeación y gestión de los territorios. En particular, las relacionadas con la sostenibilidad ambiental y la prevención de riesgos y desastres. 
6. Los programas y proyectos a desarrollar se derivan y deberán estar alineados con la visión, los objetivos estratégicos y las metas a lograr en el largo, mediano y corto plazo, definidos durante el proceso de planificación.

7. Los programas y proyectos a desarrollar encontrados durante el proceso de la planeación serán objeto de financiación con recursos del orden internacional, nacional, regional y local.

8. Por su carácter de ejecución dinámica, se requiere de una institucionalidad permanente que haga viables tanto a los programas y proyectos definidos en el documento plan, como a la aplicación de las normas establecidas en el Código de Ordenamiento Territorial.

9. Se hará explícita en el ejercicio la alineación entre el plan de ordenamiento territorial de largo plazo (a 12 años), los planes de desarrollo de corto plazo (a 4 años), los planes de inversión de la región o localidad (a 4 años) y los presupuestos anuales de inversión (Banguero, 2004).

En el plano metodológico, se ha planteado la discusión sobre si la planificación debe hacerse de arriba hacia abajo o de abajo hacia arriba, o alternativamente sobre si la planeación debe guiarse por criterios estrictamente técnicos, sin tener en cuenta las comunidades en el área de influencia, o si debe guiarse exclusivamente por la opinión comunitaria, dejando de lado la de los técnicos.

Quienes abogan por el primer enfoque -que va desde lo estratégico hasta los proyectos- opinan que las comunidades no cuentan con la suficiente información para decidir sobre las soluciones y, por lo tanto, su función se reduce a diagnosticar los problemas y proponer alternativas de solución, que luego deben pasar a ser evaluadas con la lupa de los técnicos para decidir sobre su viabilidad. En el otro extremo están quienes consideran que son las comunidades las que mejor conocen los problemas que las afectan y sus posibles soluciones, por ser quienes las viven día a día y, por lo tanto, que lo técnico debería subordinarse a la opinión comunitaria (Diez, Gutiérrez y Pazzi, 2015).

En mi opinión los dos enfoques no son mutuamente excluyentes y existen formas para hacerlos compatibles, e incluso, complementarios. En efecto, los proyectos definidos durante el ejercicio estratégico por los técnicos pueden ser confrontados con los que establecen las comunidades en la consulta y, por esta vía, llegar a un conjunto técnicamente viable y, a la vez, consensuado y apropiado por las comunidades.

Alternativamente, los técnicos podrían adelantar el ejercicio de planificación en el nivel estratégico, definiendo objetivos y estrategias gruesas, sobre todo para garantizar la consistencia del plan con las restricciones impuestas por el territorio, 
y luego definir con las comunidades los programas y proyectos requeridos para el logro de los objetivos. De esta forma, se tendría una consistencia entre lo requerido por la técnica y las demandas de las comunidades, evitando así caer en el determinismo de lo técnico o de lo comunitario (Banguero, 2001b).

Finalmente, el marco de planificación propuesto, que parte de una visión del largo hacia el corto plazo y de la macro a la micro planificación, facilita la planificación financiera de largo plazo, que puede ser desglosada posteriormente por períodos de gobierno para efectos de la búsqueda de fuentes de recursos para su ejecución, sea en el ámbito internacional, nacional, regional o local, dependiendo de la naturaleza de cada programa o proyecto. Este enfoque es conveniente para contar con información sobre los requerimientos financieros del plan completo y evitar así la ejecución a medias, por falta de recursos, de los programas o proyectos, que es algo muy frecuente en los ejercicios tradicionales de la planificación de corto plazo (Banguero, Aponte y Carrillo, 2018).

Definidos estos conceptos, principios y criterios para la elaboración de ejercicios planificación regional y urbana, se hace a continuación una aplicación de estos al caso del municipio de Buenaventura en el Valle del Cauca en Colombia.

\section{UNA APLICACIÓN: EL CASO DEL MUNICIPIO DE BUENAVENTURA}

El proceso se desarrolla en tres momentos o etapas: i) definición de la situación actual de la región o localidad (diagnóstico), ii) definición de la imagen futura deseada (visión de largo plazo de la región o localidad) y su localización en el territorio y iii) formulación de los planes estratégicos, operativos y de inversiones de desarrollo de largo y corto plazo.

Se describen a continuación los pasos a seguir en cada una de estas etapas del proceso:

\section{A. Etapa 1: construcción de la imagen de la situación actual de la región o localidad (diagnóstico)}

El proceso inicia con la consecución o construcción de mapas que reflejen adecuadamente la situación actual de la región o localidad a planificar, en términos de los siguientes componentes, listados en orden de consecución 
(Hincapié y Hoyos, 1990):

1. Mapa que contenga la morfología geográfica del territorio a planificar y de las áreas circunvecinas relacionadas.

2. Áreas de reserva ambiental. Incluyen las zonas existentes actualmente en bosques, las áreas de protección de ríos y cañadas, lagos, lagunas, costas, y los demás recursos naturales del territorio objeto del plan.

3. Áreas de alto riesgo para la localización de la población, que no son susceptibles de uso para actividades humanas (describir la situación en caso de uso para asentamientos humanos no autorizados).

4. Ríos o quebradas existentes en el territorio, zonas lacustres, y zonas costeras.

5. Concentraciones de población en ciudades y centros urbanos, diferenciados según número de habitantes en cada uno.

6. Usos actuales de los suelos disponibles (vivienda y usos residenciales urbanos, actividad económica agrícola o industrial, áreas de servicios comunales, parques, entre otros).

7. Infraestructura de vías y transporte disponibles, tanto al interior de la región o localidad, como en relación con los territorios circunvecinos. Incluye carreteras, modo ferrocarril/ acuático/aéreo, muelles, terminales de transporte de pasajeros y carga, aeropuertos, entre otros.

8. Sistemas de acueducto disponibles para abastecimiento de las comunidades y para riego en la zona rural.

9. Sistemas de alcantarillado y de disposición de aguas servidas de la región o localidad.

10. Sistema de disposición de residuos sólidos (botaderos a cielo abierto, rellenos sanitarios, plantas de tratamiento).

11. Infraestructura de servicios de salud (hospitales, clínicas, centros y puestos de salud) existentes en la región o localidad.

12. Infraestructura de servicios de educación tanto para la educación básica como para la superior.

13. Infraestructura de servicios de seguridad ciudadana y gestión de riesgos (estaciones e inspecciones de policía, cuarteles de policía y del ejército, cuarteles de bomberos, defensa civil y demás).

14. Instalaciones deportivas y otras áreas de uso para el deporte y la recreación masiva.

15. Instalaciones al servicio de la administración pública y de uso común por las organizaciones comunitarias (infraestructura del sector público).

Los Mapas 1 y 2 ilustran la localización actual de las áreas de reserva y de alto riesgo y la infraestructura de vías y transporte, respectivamente. 


\section{MAPA 1}

Situación actual de áreas de reserva forestal y de alto riesgo

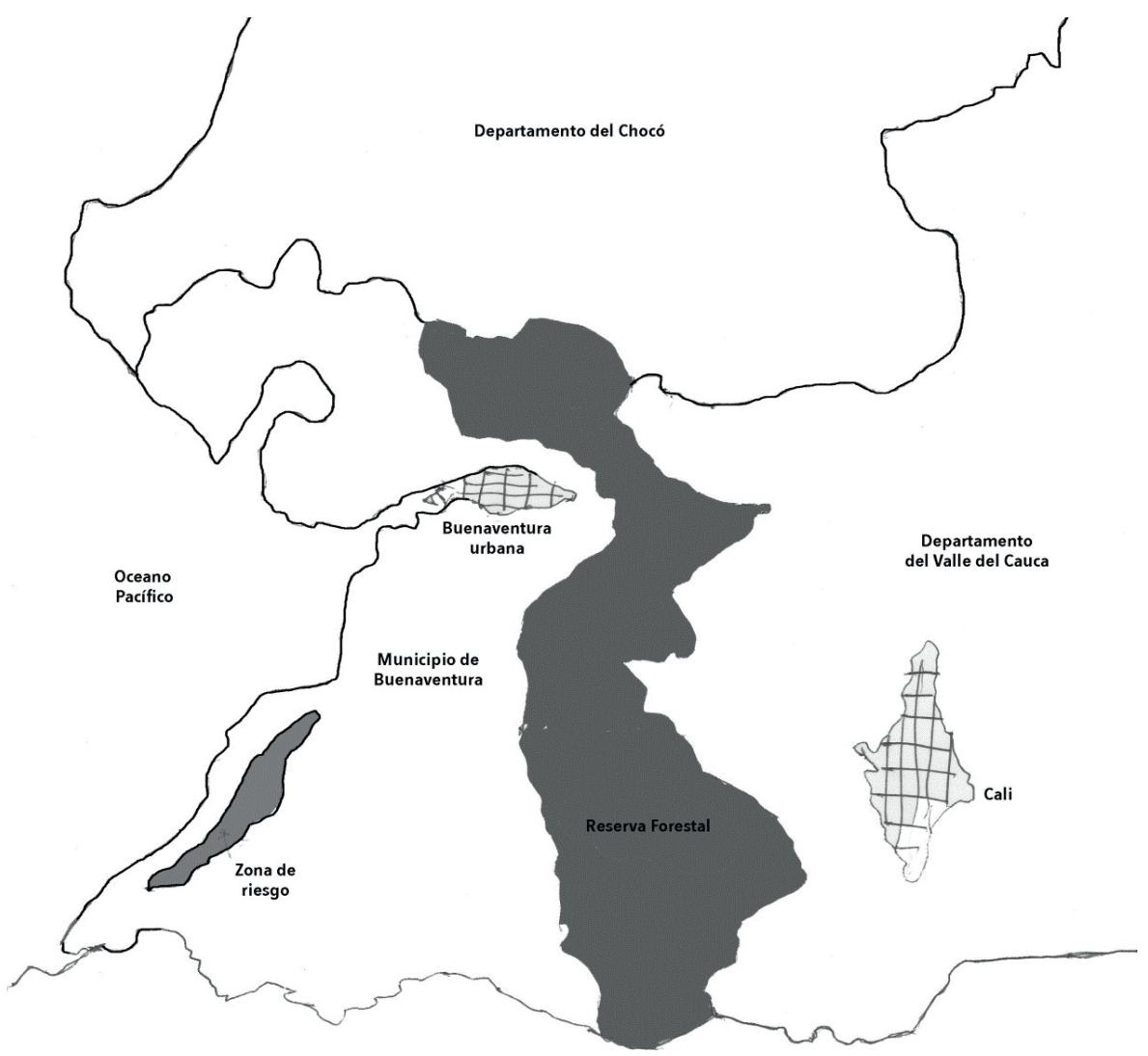

Departamento del Cauca

Fuente: Elaboración del autor.

En el Mapa 1 se presentan en color verde las áreas de reserva definidas en el plan de ordenamiento vigente y en color rojo pálido las definidas como de alto riesgo de derrumbes o inundaciones. 


\section{MAPA 2}

Situación actual de la infraestructura de vías y transporte

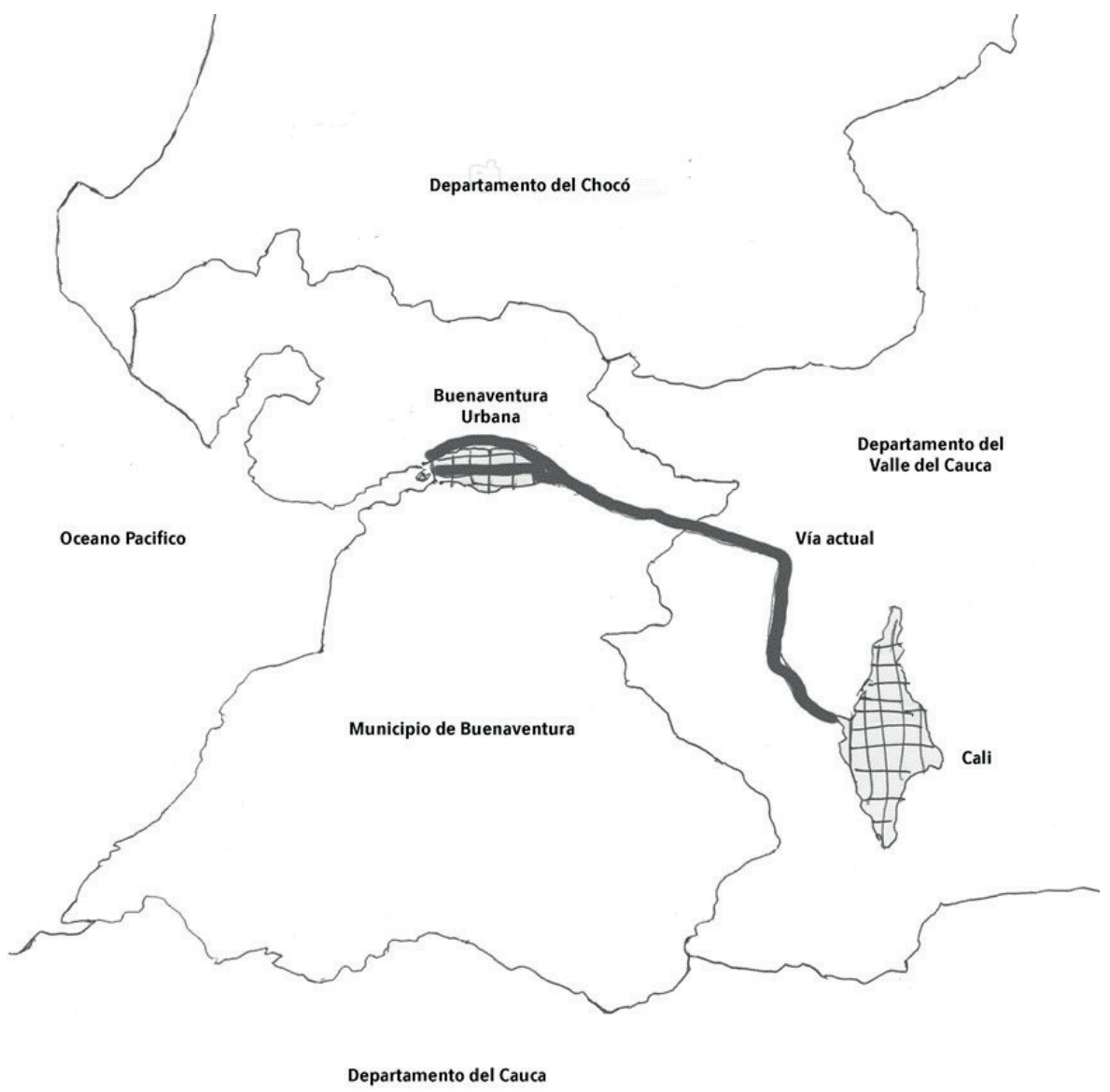

Fuente: Elaboración del autor.

En el Mapa 2 se presenta la situación actual de la vía de acceso por carretera desde Cali, capital del Departamento del Valle, hasta el puerto de Buenaventura. Nótese el acceso al área urbana por dos vías: la antigua cruzando por el centro de la ciudad y la variante por el costado occidental de la ciudad que conduce directamente al muelle en la isla Cascajal. 
Al final, es posible integrar total o parcialmente los componentes en un solo mapa (para facilitar su distinción visual se sugiere el uso de un color para cada componente).

Posteriormente, en un ejercicio de segundo nivel se pueden desagregar estos mapas, según conveniencia, para trabajar con mayor detalle, por ejemplo, centros urbanos poblados o áreas específicas en la zona rural.

Como producto de la sistematización de los resultados del ejercicio se obtiene un documento diagnóstico, el cual describe la situación actual de la región o localidad y constituye el punto de partida para la elaboración de la segunda etapa de prospectiva territorial de largo plazo.

\section{B. Etapa 2: elaboración de la visión (prospectiva) de la región o la localidad y su ubicación en el territorio}

Tras obtener la imagen actual de la región o localidad en la etapa previa, se define y construye la imagen deseada en un punto futuro en el tiempo, generalmente de largo plazo, es decir, un "sueño con ojos despiertos", que da como resultado la visión de la región o localidad expresada en las dimensiones contempladas previamente durante el diagnóstico del territorio: áreas de reserva, zonas de riesgo, usos de suelos, infraestructura de vías y transporte, infraestructura social (salud, educación, recreación y deporte, servicios públicos, entre otros) (Hincapié y Hoyos, 1990). Este ejercicio requiere necesariamente de la participación de los principales actores de la localidad en donde se realiza el plan.

\section{Definición de la visión o imagen futura deseada para la región o localidad a 12 años, según normatividad vigente para el POT (deseable: 20 años)}

Dada la diversidad de deseos y opiniones sobre el deber ser de una región o localidad, conviene emplear métodos que permitan llegar a un acuerdo sobre la imagen deseada de ella en un momento futuro previamente establecido. Para ello hay necesidad de convocar, presencial o virtualmente, a los actores de la región o localidad, para que, mediante métodos como la lluvia de ideas (de identificación) y el ábaco de Regnier (de selección) se llegue a su definición consensuada (Mojica, 1991, 2005; Godet, 1995; Ortegón y Medina, 1997; y Banguero, 2001a). 
Para lograrlo se sugiere el siguiente procedimiento:

1. Elaborar un listado de actores representativos de la región o localidad, teniendo cuidado de incluir a representantes del sector público y privado, ONGs, y comunidades. Dado que se trata de lograr un consenso sobre lo fundamental de la visión, es importante para un mayor compromiso ulterior que haya una participación lo más amplia posible de los líderes de la región o localidad.

2. Hacer llegar a todos los actores una síntesis de los principales resultados obtenidos en el ejercicio previo de definición de la situación actual (Diagnóstico).

3. Adelantar un ejercicio de lluvia de ideas, solicitando a cada uno de los actores, que defina, de forma presencial o virtual, los elementos que, a partir del diagnóstico enviado y su visión sobre futuro de la región o localidad, deben ser considerados en la definición de la imagen futura o visión al final del período establecido, teniendo en cuenta al menos cuatro dimensiones del desarrollo: económica, social, ambiental e institucional de una región o localidad (CAF, 2010; y Banguero, 2011, 2017).

En la dimensión económica se consideran aspectos del ordenamiento territorial relacionados con infraestructura de vías y transporte y suelos disponibles para usos agropecuarios o industriales.

En la dimensión social se consideran aspectos del ordenamiento territorial relacionados con el perímetro urbano, las áreas para vivienda residencial, la infraestructura de servicios públicos de acueducto, alcantarillado, energía, y gas, la disposición de residuos líquidos y sólidos, la infraestructura de salud, educación, recreación y deportes, cultura, y los parques y zonas comunales.

En la dimensión ambiental se consideran aspectos del ordenamiento territorial como las áreas de reserva forestal, las zonas de alto riesgo por derrumbes o inundaciones, y las zonas de protección de ríos y cañadas, lagos y costas.

En la dimensión institucional se contemplaron aspectos del ordenamiento territorial relacionados con la infraestructura del sector público y la infraestructura de seguridad ciudadana (i.e. estaciones de policía, ejército, bomberos, defensa civil).

Los formatos elaborados previamente que contengan las posibles opciones pueden ser útiles para guiar a los participantes en el ejercicio.

4. Recogidas las sugerencias, se tabulan y se compila un texto con los componentes propuestos por los actores para ser incluidos en la posible visión e imagen futura deseada de la región o localidad. 
5. La selección del texto definitivo se hace aplicando el método del Ábaco de Regnier, en el que cada participante expresa su grado de acuerdo o desacuerdo con cada uno de los componentes propuestos para la visión y las razón de su posición. Al final, se construye un texto que contenga todos aquellos componentes sobre los cuales existió consenso o, al menos, una opinión mayoritaria cualificada (dos tercios o más de los actores). Este texto deberá recibir al final la aprobación de todos los participantes en el ejercicio (Mojica, 1990, 2005; y Banguero, 2001a).

Luego de estar definida la visión, se deberá hacer un ejercicio de localizar en el territorio lo que se considere pertinente. Esto se puede hacer sobre los mismos mapas que se usaron para describir la situación actual (mapas diagnóstico), teniendo el cuidado de diferenciar lo nuevo de lo que ya existe, por ejemplo, señalándolo con notación puntuada o, alternativamente, construir nuevos mapas con sólo la proyección de lo nuevo a desarrollar en cada componente hacia el futuro, conservando la notación de colores usada en los mapas de diagnóstico. Al final, es posible indicar en un solo mapa varios o la totalidad de los componentes definidos en el POT, para verificar su consistencia.

Los Mapas 3 y 4 ejemplifican este procedimiento para las áreas de reserva y de protección de riesgos y la infraestructura de vías y transporte, respectivamente.

En el Mapa 3 se propone la ampliación de la actual zona de reserva forestal para adicionar la franja contigua, entre la alta montaña y la planicie del litoral, y, adicionalmente, la inclusión de una nueva zona de riesgo en la parte norte del municipio, en los límites con el departamento del Chocó.

En el Mapa 4 se propone la construcción de una nueva vía de acceso a la isla de Cascajal por el lado oriental del casco urbano (indicado en amarillo en el mapa), para mejorar el acceso a la isla y la construcción de avenidas transversales de oriente a occidente en el área urbana localizada en el continente para mejorar la movilidad interna en el área urbana del territorio continental. Adicionalmente, se propone la construcción de un moderno ferrocarril, de doble vía, trocha ancha y electrificado, desde Cerrito, en el centro del Valle hasta Buenaventura, construyendo la variante Mulaló - Loboguerrero como una alternativa más económica para el transporte de la carga desde el Centro del país hasta Buenaventura. Finalmente, la propuesta incluye la construcción de un moderno muelle de contenedores en alta mar, localizado antes de la entrada a la bahía, con el propósito de hacer el cargue y descargue de barcos de gran calado, que hoy tienen dificultades para acceder a la bahía por su limitada profundidad. 


\section{MAPA 3}

Imagen futura deseada de áreas de reserva forestal y de zonas de alto riesgo

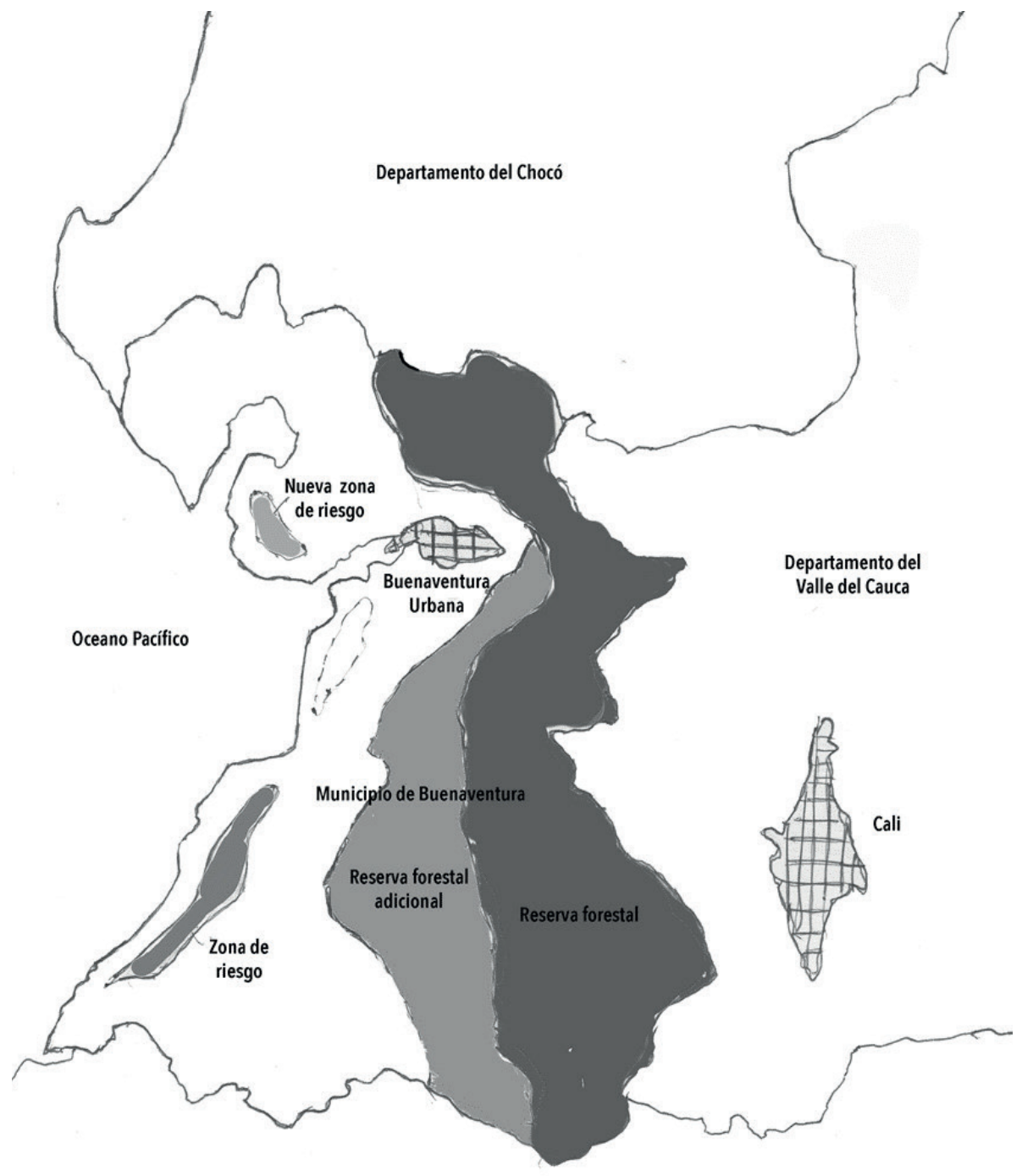

Departamento del Cauca

Fuente: Elaboración del autor. 


\section{MAPA 4}

Imagen futura deseada de la infraestructura de vías y transporte

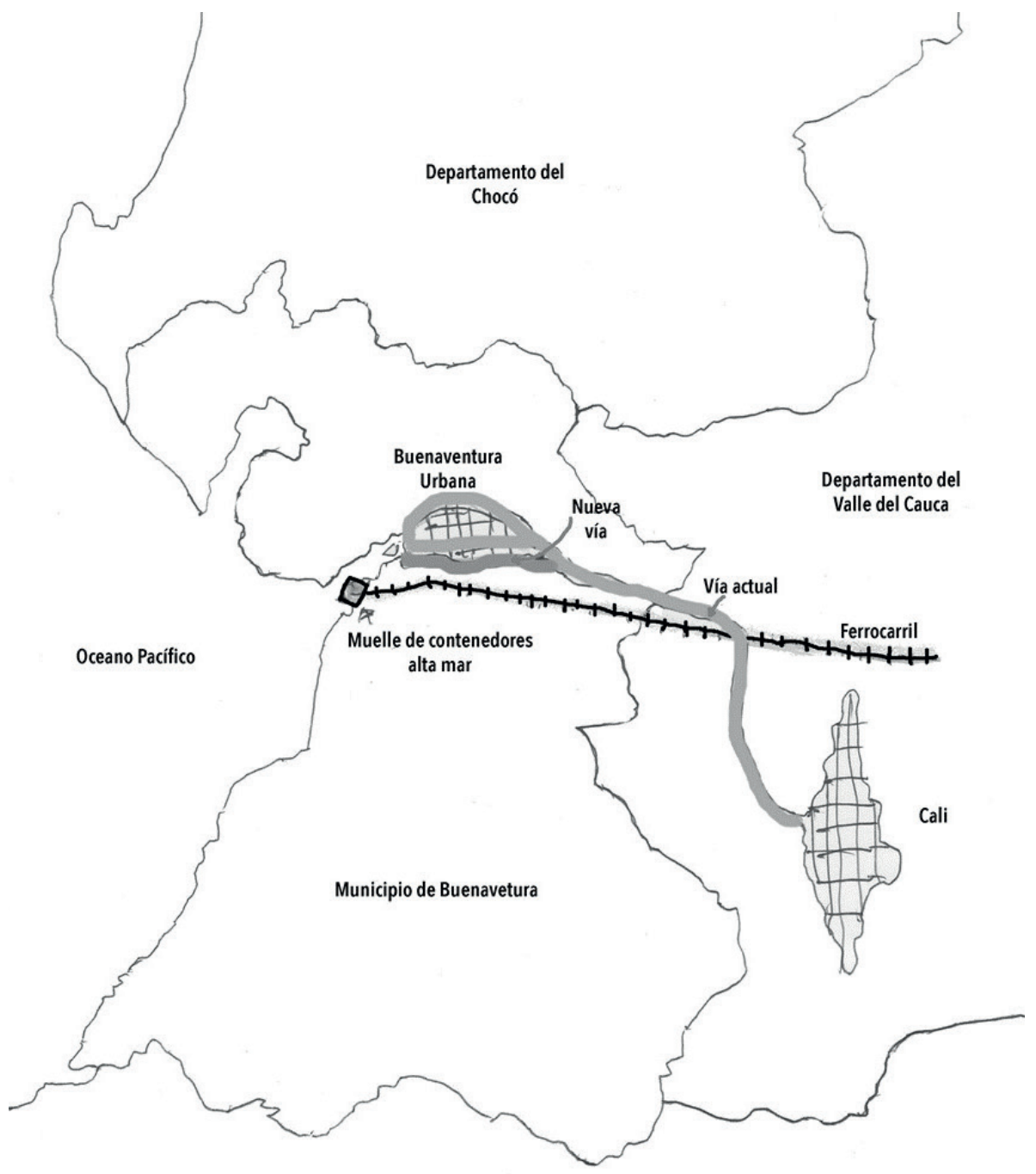

Departamento del Cauca

Fuente: Elaboración del autor. 
Esta etapa genera dos productos. Primero, el texto de la visión o imagen futura deseada para la región o localidad al final del período de la planeación (12 años en la actualidad para el POT). Y segundo, el plan de ordenamiento territorial de largo plazo, reflejado visualmente en la cartografía construida y en un texto contentivo de las normatividad a establecer para hacerlo cumplir (borrador del Código de Ordenamiento Territorial a 12 años, según la normatividad vigente actualmente en Colombia). ${ }^{1}$

D. Etapa 3: elaboración de los planes estratégicos y operativos regionales o municipales de largo y corto plazo

El trabajo desarrollado en la etapa anterior es un insumo fundamental para la elaboración de los planes estratégicos y operativos (programas y proyectos) de largo y corto plazo (Sache, 1989; Pérez, 1990; y Banguero, 2001a) para la región o localidad objetivo (Gráfico 2).

\section{GRÁFICO 2}

El diagnostico, la visión y el plan estratégico: esquema de interrelaciones

$\underline{\text { Diagnóstico }}$

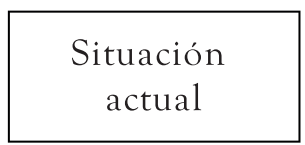

$\underline{\text { Visión }}$

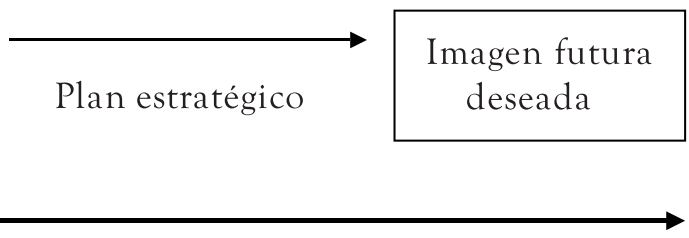

\section{Tiempo}

Fuente: Elaboración del autor.

Para efectos de la definición del componente estratégico de los planes, los objetivos definidos en la visión de largo plazo se clasifican en cuatro grandes dimensiones: la ambiental, la social, la económica y la institucional (CAF, 2010; y Banguero, 2011, 2017).

\footnotetext{
${ }^{1}$ Deseable a 20 años.
} 


\section{E. El plan estratégico de largo plazo (12 años según normatividad actual para el POT)}

A partir de la visión definida en la etapa anterior se definen los objetivos estratégicos, las metas y las estrategias requeridas para el logro de la visión e imagen futura deseada, en todas sus dimensiones al final del período de 12 años (contemplado por la normatividad vigente sobre el POT), originando el Plan Estratégico Regional o Local de largo plazo (Cuadro 1).

\section{CUADRO 1}

Estructura básica de un plan estratégico

\begin{tabular}{|c|c|c|}
\hline \multicolumn{2}{|c|}{ Fin o razón de ser de la institución } & $\begin{array}{c}\text { Misión y principios } \\
\text { institucionales }\end{array}$ \\
\hline \multicolumn{2}{|c|}{ Imagen futura deseada y factible } & Visión institucional \\
\hline Objetivo 1 & Objetivo 2 & Objetivos estratégicos \\
\hline Estrategia 1 & Estrategia 2 & \\
Política 1.1 & Política 2.1 & \\
Política 1.2 & Política 2.2 & Estrategias \\
" & " & \\
" & " & \\
Política 1.n & Política 2.n & \\
\hline
\end{tabular}

Fuente: Tomado de Banguero (2001, página 98).

Para orientación del lector, se ilustra la elaboración del plan con el siguiente ejemplo:

\section{Dimensión ambiental}

Objetivo estratégico: Construcción de una región o localidad ambientalmente sostenible. En esta dimensión se contemplan aspectos tales como la forestación, la minería, la erosión y contaminación de suelos, la contaminación del agua 
y del aire, y la prevención de desastres naturales. El Cuadro 2 presenta un esquema de indicadores de logros y metas para este objetivo.

\section{CUADRO 2}

Indicadores y metas para el objetivo sostenibilidad ambiental

\begin{tabular}{|l|c|}
\hline \multicolumn{1}{|c|}{ Indicador de logro } & Meta \\
\hline Kilómetros cuadrados de bosque natural conservado & \\
\hline Toneladas de residuos sólidos adecuadamente dispuestos & \\
\hline Metros cúbicos de agua tratada & \\
\hline Índice de contaminación aérea & \\
\hline
\end{tabular}

Fuente: Elaboración del autor.

Estrategias: ${ }^{2}$

a. Definir en el POT áreas de reserva forestal y de protección de riesgos, y velar por su cumplimiento.

b. Construir la infraestructura adecuada para garantizar la disposición correcta de los residuos sólidos (e.g. rellenos sanitarios, plantas de reciclaje).

c. Construir la infraestructura necesaria para garantizar el tratamiento adecuado de las aguas residuales (e.g. plantas de tratamiento).

\section{Dimensión social}

Objetivo estratégico: Construcción de una región o localidad socialmente sostenible. En esta dimensión se contemplan aspectos tales como la nutrición, la salud, la vivienda, los servicios básicos, la educación, la recreación, el deporte, la cultura, el empleo, la seguridad social, la seguridad ciudadana y demás aspectos relacionadas con la inversión social y la creación de capital humano. El Cuadro 3 presenta un esquema de indicadores de logro y metas para este objetivo.

\footnotetext{
${ }^{2}$ Hace referencia al cómo lograrlo, o sea a las políticas a seguir en cada dimensión para el logro de las metas y el objetivo estratégico.
} 


\section{CUADRO 3}

Indicadores y metas para el objetivo de sostenibilidad social

\begin{tabular}{|l|l|}
\hline \multicolumn{1}{|c|}{ Indicador de logro } & Meta \\
\hline Tasa de desnutrición infantil & \\
\hline Tasa de mortalidad infantil & \\
\hline Tasa de mortalidad general & \\
\hline Tasa de analfabetismo & \\
\hline Tasa de desempleo abierto & \\
\hline Cobertura de la educación media básica & \\
\hline Cobertura del servicio de acueducto - alcantarillado & \\
\hline
\end{tabular}

Fuente: Elaboración del autor.

Estrategia:

a. Desarrollar programas de atención a la primera infancia priorizando a las madres embarazadas y a los niños en condición de vulnerabilidad nutricional.

b. Universalizar la educación preescolar (i.e. prejardín, jardín y transición) para la población de cuatro a seis años de edad.

c. Mejorar la cobertura y reducir los niveles de deserción de la educación media (primaria y bachillerato).

d. Ampliar la cobertura de la educación superior en los niveles técnico, tecnológico y profesional, al igual que en programas de postgrado.

e. Desarrollar programas de vigilancia epidemiológica para garantizar la inmunización de la totalidad de la población entre cero y cinco años.

f. Mejorar los sistemas de servicios de salud, para garantizar atención oportuna y de calidad para toda la población.

g. Desarrollar programas de construcción y mejoramiento de vivienda para dotar de vivienda digna a la totalidad de la población.

h. Mejorar los sistemas de suministro de agua potable y de evacuación y tratamiento de las aguas servidas en el área urbana y los núcleos poblados en el sector rural.

i. Adelantar programas de fomento a la recreación, el deporte y la cultura con acceso a toda la población. 
j. Diseñar y poner en marcha un plan de incentivos para la generación de empleos productivos y remunerativos para la totalidad de la población económicamente activa en las áreas urbanas y rurales de la región o localidad.

k. Ampliar la vinculación de la totalidad de la, población a los sistemas de seguridad social y pensiones.

1. Mejorar la eficiencia y la eficacia de los organismos de la policía y la justicia en la lucha contra la delincuencia.

\section{Dimensión económica}

Objetivo estratégico: Crecimiento económico sostenible, con equidad y generación de empleo. En esta dimensión se consideran aspectos como la producción sostenible en el campo, la industrialización con generación de empleos productivos y remunerativos, el turismo, el comercio, los servicios, y el comercio exterior. El Cuadro 4 presenta un esquema de indicadores de logro y metas para este objetivo.

\section{CUADRO 4}

Indicadores y metas para el objetivo sostenibilidad económica

\begin{tabular}{|l|l|}
\hline \multicolumn{1}{|c|}{ Indicador de logro } & Meta \\
\hline Tasa de crecimiento de la producción total & \\
\hline Tasa de crecimiento de la producción agrícola & \\
\hline Tasa de crecimiento de la producción industrial & \\
\hline Porcentaje de ingreso del 20\% más pobre de la población & \\
\hline Tasa de desempleo abierto & \\
\hline
\end{tabular}

Fuente: Elaboración del autor.

Estrategia:

a. Diseñar y poner en marcha un plan de incentivos y apoyos para los pequeños productores del sector agropecuario de la región o localidad.

b. Diseñar e implementar un plan de incentivos y apoyos para el desarrollo 
de la pequeña y la mediana industria en la región o localidad, en especial la generadora de empleo.

c. Diseñar y poner en marcha un plan de construcción o mejoramiento de las vías secundarias y terciarias de región o localidad para facilitar el transporte de alimentos de las áreas rurales a la urbana.

d. Diseñar e implementar un plan de fomento y apoyo a las actividades turísticas y culturales de la región o localidad.

\section{Dimensión institucional}

Objetivo estratégico: Construcción de una institucionalidad sostenible, eficiente y eficaz. En esta dimensión se contemplan aspectos como la eficacia de las entidades públicas, la eficiencia en los procesos de atención al público, la agilidad en los procesos de contratación, la ética en el manejo de los recursos públicos, la eficiencia de las autoridades responsables de la seguridad ciudadana, la eficacia de los organismos encargados de la justicia, el grado de organización de las comunidades y la eficacia de las organizaciones comunitarias, entre otros. El Cuadro 5 presenta un esquema de indicadores de logro y metas para este objetivo.

\section{CUADRO 5}

Indicadores y metas para el objetivo sostenibilidad institucional

\begin{tabular}{|l|l|}
\hline \multicolumn{1}{|c|}{ Indicador de logro } & Meta \\
\hline Tiempo de espera (promedio de días) en trámite público & \\
\hline Tasa de delitos contra el erario público & \\
\hline Tasa de homicidios por 100.000 habitantes & \\
\hline Productividad media por trabajador público & \\
\hline Tasa de evasión en impuestos y contribuciones & \\
\hline Número de organizaciones comunitarias & \\
\hline Gasto social como porcentaje del gasto total & \\
\hline
\end{tabular}

Fuente: Elaboración del autor. 


\section{Estrategia:}

a. Fortalecer la planeación estratégica y operativa en todos los niveles de la administración pública, para mejorar la eficiencia y la asignación de los recursos públicos y su eficacia en términos de resultados.

b. Adelantar un programa permanente de cualificación de funcionarios públicos a todos los niveles de la administración, para elevar su productividad.

c. Revisar y simplificar las estructuras organizacionales, los procesos y procedimientos en las entidades del sector público, para hacerlos más eficientes y eficaces.

d. Fortalecer los mecanismos de control y veeduría ciudadana para ejercer una adecuada vigilancia en el uso de los recursos públicos y su correcta aplicación.

e. Fortalecer las organizaciones comunitarias en su labor de gestión y fiscalización de sus proyectos públicos.

\section{F. Plan operativo de largo plazo (12 años)}

Constituye la otra cara de la moneda del plan estratégico y en él se consignan los programas y proyectos a desarrollar en los doce años para dar cumplimento a cada uno de los objetivos del plan estratégico.

Este ejercicio requiere generalmente de la participación de los actores de la región o localidad en la escogencia de los programas y proyectos, y de los técnicos para su diseño. La metodología del marco lógico, ampliamente usada por entidades como el Banco Mundial o las Naciones Unidas en la gestión de proyectos de desarrollo, puede ser muy útil para estructurar este componente del plan (Banguero, 2001b; y Aldunate y Córdoba, 2011). Muchos de los programas y proyectos ya fueron previamente reconocidos durante el ejercicio de elaboración del POT a largo plazo y sólo se necesita retomarlos en este plan.

Para efectos de la alineación de los programas y proyectos con los objetivos estratégicos se desarrolla un esquema matricial ubicando en sentido horizontal los objetivos estratégicos del plan y en sentido vertical el nombre de los programas y proyectos que se esperan desarrollar para el logro de cada objetivo estratégico. En esta matriz sólo aparece el enunciado de cada programa o proyecto (Cuadro 6) .

El detalle de cada proyecto en términos de su localización, objetivos, actividades, cronograma, responsable, presupuesto y demás aspectos que se consideren necesarios, debe señalarse explícitamente en la matriz del marco lógico a elaborar para cada programa o proyecto incluido en el plan operativo 
de largo plazo. Un resultado importante de la especificación de cada programa o proyecto incluido en el esquema del marco lógico es su valoración económica, a partir de la definición de los recursos humanos y físicos requeridos para la ejecución de cada una de las actividades del programa o proyecto y sus costos unitarios.

\section{CUADRO 6}

Alineación del plan estratégico con el plan operativo

\begin{tabular}{|c|c|c|c|c|}
\hline \multirow{3}{*}{$\begin{array}{c}\text { Componente } \\
\text { Estratégico }\end{array}$} & \multicolumn{4}{|c|}{ Misión } \\
\hline & \multicolumn{4}{|c|}{ Visión } \\
\hline & \multicolumn{2}{|c|}{$\begin{array}{l}\text { Objetivo } 1 \\
\text { Estrategia } 1\end{array}$} & \multicolumn{2}{|c|}{$\begin{array}{c}\text { Objetivo } 2 \\
\text { Estrategia } 2\end{array}$} \\
\hline \multirow{8}{*}{$\begin{array}{c}\text { Componente } \\
\text { Operativo }\end{array}$} & Programa 1.1 & Programa 1.2 & Programa 2.1 & Programa 2.2 \\
\hline & Proyecto 1.1.1 & Proyecto 1.2.1 & Proyecto 2.1.1 & Proyecto 2.2 .1 \\
\hline & Proyecto 1.1.2 & Proyecto 1.2 .2 & Proyecto 2.1.2 & Proyecto 2.2 .2 \\
\hline & Proyecto 1.1.3 & Proyecto 1.2 .3 & Proyecto 2.1.3 & Proyecto 2.2 .3 \\
\hline & “ & “ & “ & “ \\
\hline & “ & “ & “ & “ \\
\hline & “ & “ & “ & “ \\
\hline & Proyecto 1.1.n & Proyecto 1.2.n & Proyecto 2.1.n & Proyecto 2.2.n \\
\hline
\end{tabular}

Fuente: Banguero (2001, p. 110).

\section{G. Plan de inversiones de largo plazo (12 años)}

La estimación de los costos de cada programa o proyecto incluido en el plan operativo permite la elaboración del correspondiente plan de inversiones a lo largo de los 12 años. Generalmente se presenta en un esquema matricial, en donde se describan los programas y proyectos de forma vertical y sus costos de manera horizontal (Cuadro 7). 


\section{CUADRO 7}

Matriz de un plan plurianual de inversiones de largo plazo

\begin{tabular}{|c|c|c|c|c|c|c|c|c|c|c|c|c|c|}
\hline $\begin{array}{c}\text { Programa/ } \\
\text { Proyecto }\end{array}$ & 1 & 2 & 3 & 4 & 5 & 6 & 7 & 8 & 9 & 10 & 11 & 12 & Total \\
\hline $\begin{array}{l}\text { Objetivo } 1 \\
\text { Programa } 1.1\end{array}$ & & & & & & & & & & & & & \\
\hline Proyecto 1.3 & & & & & & & & & & & & & \\
\hline $\begin{array}{l}\text { Objetivo } 2 \\
\text { Programa } 2.1\end{array}$ & & & & & & & & & & & & & \\
\hline Proyecto 2.3 & & & & & & & & & & & & & \\
\hline $\begin{array}{l}\text { Objetivo } 3 \\
\text { Programa } 3.1\end{array}$ & & & & & & & & & & & & & \\
\hline Proyecto 3.3 & & & & & & & & & & & & & \\
\hline $\begin{array}{l}\text { Objetivo } 4 \\
\text { Programa } 4.1\end{array}$ & & & & & & & & & & & & & \\
\hline Proyecto 4.3 & & & & & & & & & & & & & \\
\hline Total & & & & & & & & & & & & & $\begin{array}{l}\text { Gran } \\
\text { total }\end{array}$ \\
\hline
\end{tabular}

Fuente: Elaboración del autor.

\section{H. Planes de corto plazo (períodos de 4 años)}

Una vez estructurado el plan de desarrollo de largo plazo (a 12 años) y los correspondientes planes operativo y de inversiones, la tarea siguiente es fraccionarlos en períodos de cuatro años, haciéndolos corresponder con los períodos de los gobernantes regionales o locales. La forma de hacerlo es la siguiente:

En lo concerniente al plan estratégico, se definen metas intermedias a lograr 
en cada uno de los períodos de cuatro años de gobierno, de tal forma que al final se alcance la meta total estipulada para los 12 años, es decir, la correspondiente a la imagen futura deseada en cada componente.

Un ejemplo ayuda a clarificar el proceso. Supongamos que en lo relacionado con la cobertura de la educación preescolar la meta al final el período de 12 años es del $100 \%$ de la población en edad de cuatro a seis años. Si la tasa de cobertura actual es del $40 \%$, podemos definir como meta a lograr al final del primer período de cuatro años el $60 \%$, del $80 \%$ al finalizar el segundo período y lógicamente del 100\% al finalizar el período de los 12 años.

Este arreglo gradual se repite con todas las metas definidas en el plan estratégico de largo plazo, originando así tres planes estratégicos para sendos períodos de gobierno responsables de la ejecución del plan de ordenamiento territorial a 12 años.

El paso siguiente es la selección de los programas y proyectos a desarrollar en cada período, de tal forma que su ejecución garantice el logro de las metas intermedias al finalizar cada período de gobierno. En algunos proyectos -no ejecutables en su totalidad en un período- debe estipularse la parte que corresponde a cada período de gobierno, de tal forma que se garantice su ejecución completa al finalizar el período del POT (12 años). El marco lógico y la matriz de inversiones (elaborada en el Cuadro 3) son de utilidad en este propósito.

La valoración de programas y proyectos seleccionados para ejecutar en cada período de gobierno, permiten la elaboración del plan plurianual de inversiones para cada período de cuatro años y su incorporación en los presupuestos anuales de inversión.

Como resultado de los ejercicios realizados en la tercera etapa se generan dos productos. Primero, unos Planes estratégicos, operativos y de inversiones de largo plazo (12 años), alineados y consistentes con el POT elaborado para el mismo período. Y segundo, unos Planes estratégicos, operativos y de inversiones para períodos de cuatro años (corto plazo), alineados y consistentes con el Plan Estratégico de Desarrollo de largo plazo y su correspondiente POT (a 12 años).

\section{El sistema de información para el monitoreo y la evaluación de los resultados}

El desarrollo del proceso descrito en este trabajo requiere de la existencia o configuración de un sistema de información tal que permita la construcción de la línea de base para el diagnóstico, o situación actual, el monitoreo del avance 
logrado en cada una de las etapas (cada cuatro años) y la evaluación de los resultados al final del horizonte del plan (12 años).

Para cumplir con este propósito es deseable y recomendable estructurar y poner en marcha un observatorio para supervisar la marcha del proceso en la región o localidad objetivo, instrumento fundamental para garantizar una oportuna rendición de cuentas a la comunidad. El diseño de este sistema de información debe estar alineado con la visión, los objetivos estratégicos, las metas, los programas y proyectos y el plan de inversiones, definidos en los planes de largo y corto plazo (Banguero, 2001a).

\section{J. La viabilidad política de la planeación estratégica territorial}

Para hacer viable este proceso de planificación de largo plazo, se esperaría que los gobernantes de turno tomen como referente para la elaboración de su programa de gobierno los lineamientos definidos para cada período de gobierno en este ejercicio, de tal forma que ello garantice el logro de la imagen futura deseada (visión) para la región o localidad en el largo plazo (12 años).

Existen dos alternativas para lograr este propósito. Una es la permanencia en el gobierno municipal de un grupo político que garantice su cumplimiento en el largo plazo. La otra es que las fuerzas vivas de la región o localidad se organicen para actuar como garantes de hacer cumplir el plan en el horizonte de los tres períodos de gobierno implicados, reduciendo así las posibilidades de su incumplimiento.

\section{CONCLUSIONES}

Históricamente los ejercicios de planificación han tenido momentos diferentes en la dimensión tiempo y espacio. En efecto, la revisión de la literatura sobre el tema muestra que las preocupaciones sobre la necesidad de planificar el espacio se iniciaron a principios del siglo XIX y se consolidaron el siglo XX, en tanto que la planeación estratégica de largo plazo es un hecho relativamente reciente en la historia de la humanidad. Esta es, quizá, la explicación del hecho de que los dos tipos de planeación han corrido en paralelo, tanto en su normatividad como en la práctica. En el caso colombiano, esta dicotomía está reflejada en el hecho de que existen dos leyes, completamente independientes y expedidas en años diferentes, para regular lo relacionado con la planeación 
regional y urbana en el tiempo y el espacio.

En este trabajo se presentan argumentos para afirmar que estos dos tipos de planeación están estrechamente relacionados en el contexto regional y urbano y que, por lo tanto, hay necesidad de integrarlos para hacerlos consistentes, dado que la planificación en el tiempo requiere de un ejercicio previo de planificación del espacio en el largo plazo, el cual actúa como restricción al logro de las metas en el tiempo. El esfuerzo de integración parte de la formulación de dos principios básicos: la planeación en el tiempo va del largo al corto plazo, en tanto que la planeación del espacio se hace de la macro a la micro planificación. Adicionalmente, se retoman algunos criterios definidos por expertos en planificación local para hacer viable el desarrollo del proceso.

En este trabajo también se incluyó una aplicación detallada de este enfoque, usando el caso del municipio de Buenaventura, en el Valle del Cauca, Colombia. Con esta aplicación se pretendió enseñar la racionalidad teórica y metodológica de un ejercicio de planificación en el cual se integran de manera consistente los componentes básicos de un plan estratégico (diagnóstico, visión, objetivos y metas) en el tiempo, con la localización en el espacio de los programas y proyectos de usos del suelo y construcción de la infraestructura requerida para lograr los objetivos, las metas, y la visión e imagen futura deseada, de una región o localidad. Esta integración se hizo en un horizonte de tiempo de largo plazo, y en la doble dimensión espacio-tiempo, sugiriendo así su viabilidad técnica y social. Queda en manos de los gobernantes el hacerla políticamente viable.

\section{REFERENCIAS}

Aldunate, Eduardo y Córdoba, Julio (2011), Formulación de Programas con la Metodología del Marco Lógico. Santiago de Chile. Serie Manuales, 68. Naciones Unidas. CEPAL.

Banguero, Harold (1982), "Colombia 2000: A Framework for Population, Employment, Growth, Income Distribution and Essential Human Needs Planning”. Geneva. ILO Working Paper WEP 2-32fVVP 31.

Banguero, Harold (1985), "Colombia Basic Needs Model. Colombia 2000. Versión II”. Geneva. Informe OIT.

Banguero, Harold (2001a), Prospectiva y planeamiento estratégico. Un enfoque aplicado. Cali. Colombia. Cali: Editorial Facultad de Humanidades. Universidad del Valle.

Banguero, Harold (2001b), Gerencia integral de proyectos: Un enfoque de ciclo de 
vida. Cali. Colombia. Cali: Editorial Facultad de Humanidades, Univalle.

Banguero, Harold (2004), Teoría y Práctica de la Gestión Municipal. Cali: Editorial Facultad de Humanidades. Universidad del Valle.

Banguero, Harold (2011), "Haciendo realidad el desarrollo humano sostenible," en Revista de Economía y Administración, Vol. 9, No. 2.

Banguero, Harold (2017), Haciendo sostenible el desarrollo Invirtiendo la causalidad: hacia el desarrollo económico por el desarrollo social. Berlín: Editorial Académica Española.

Banguero, Harold., Carrillo, Lilian y Aponte, Elizabeth (2018), Planeación y gestión estratégica de las organizaciones: conceptos, métodos y casos de aplicación. Cali: Editado por la Universidad Autónoma de Occidente.

Berger, Gaston (1964), Phenomenologie du temps et propospective. París: Presses Universitatres de France.

Boisier, Sergio (1999), Teorías y metáforas sobre el desarrollo territorial, Santiago de Chile: CEPAL.

Boisier, Sergio (2006), "Algunas reflexiones para aproximarse al concepto de ciudad-región", Ánfora. Revista Científica de la Universidad Autónoma de Manizales. Vol. 13. No. 21.

CAF (Corporación Andina de Fomento) (2010), Desarrollo Local: Hacia un nuevo protagonismo de las ciudades y regiones. Caracas: Reporte de Economía y Desarrollo.

Congreso de la República de Colombia (1993), Ley 152 de 1993, sobre la planeación y el plan de desarrollo. Bogotá. Colombia.

Congreso de la República de Colombia (1997), Ley 388 de 1997, sobre el ordenamiento territorial.

De Jouvenel, Bertrand (1964), The Art of Conjecture. New York: Basic Books.

Diez, José, Ricardo Gutiérrez y Andrés Pazzi. (2015), “¿De arriba hacia abajo o de abajo hacia arriba? Un análisis crítico de la planificación del desarrollo en América Latina", Geopoliticas. Revista de Estudios sobre Espacio y Poder. Vol. 4. No.2.

Fujita, Masahisa (2000), Economía Espacial. Barcelona: Editorial Ariel.

Godet, Michel (1985), Prospective et planification strategique. París: Económica.

Godet, Michel (1995), De la anticipación a la acción. Manual de prospectiva y estrategia. México D.F.: Editorial Alfa Omega.

Godet, Michel (1997), Prospectiva. ¿Por qué? ¿Cómo ? Siete claves, en Edgard Ortegón y Javier Medina, compiladores. Prospectiva: construcción social del futuro. Cali: Universidad del Valle- ILPES.

Hincapié, Martha Lucía y Hoyos, Gonzalo (1990), El Plan municipal. Quito: 
Manual publicado por IULA- CELCADEL (Centro Latinoamericano de Capacitación y Desarrollo de los Gobiernos Locales).

Isard, Walter (1956), Location and space economy: a general theory relating to industrial location, market areas and trade, land use, trade and urban structure. Cambridge, MA: MIT y Wiley.

Krugman, Paul (1991), "Increasing returns and economic geography", Journal of Political Economy, 99, 3.

Losch, August. (1957), Teoría económica espacial. Buenos Aires: Editorial Ateneo.

Matarrese, José Emilio (1969), La teoría económica espacial en la historia del pensamiento económico. Tesis doctoral. Facultad de Ciencias Económicas. Buenos Aires: Universidad de Buenos Aires.

Mojica, Francisco José (1991), La prospectiva. Técnicas para visualizar el futuro. Bogotá: Editorial Legis.

Mojica, Francisco José (2005), La construcción de futuro: Concepto y modelo de prospectiva estratégica, territorial y tecnológica. Bogotá: Editado por el Convenio Andrés Bello y la Universidad Externado de Colombia.

Ortegón, Edgar y Medina, Javier (compiladores) (1997), Prospectiva. Construcción Social del Futuro. Cali: Editado por ILPES y Facultad de Administración, Universidad del Valle.

Pérez, Martha (1990), Guia práctica de planeación estratégica. Cali: Editorial Facultad de Administración, Universidad del Valle.

Perroux, Francois (1964), La economía del siglo XX. Barcelona: Editorial Ariel.

Sache. Matthias (1989), "Bases para el diseño e implantación de un sistema de planificación estratégica”. Santiago de Chile.: CLAD, Serie Temas de Coyuntura en Gestión Pública.

Sallenave, Jean Paul (1985), Gerencia y planeación estratégica. Cali: Editorial Norma.

Von Thunen, Johann (1910), Der Isolierte State. Berlín: Jega y Fiacher. 